\title{
Smooth transonic flow in an array of counter-rotating vortices
}

\author{
By G. K. O’REILLY†AND D. I. PULLIN \\ Graduate Aeronautical Laboratories, 105-50, California Institute of Technology, \\ Pasadena, CA 91125, USA
}

(Received 28 July 2004 and in revised form 7 October 2004)

Numerical solutions to the steady two-dimensional compressible Euler equations corresponding to a compressible analogue of the Mallier \& Maslowe (Phys. Fluids, vol. A 5, 1993, p. 1074) vortex are presented. The steady compressible Euler equations are derived for homentropic flow and solved using a spectral method. A solution branch is parameterized by the inverse of the sound speed at infinity, $c_{\infty}^{-1}$, and the mass flow rate between adjacent vortex cores of the corresponding incompressible solution, $\epsilon$. For certain values of the mass flux, the solution branches followed numerically were found to terminate at a finite value of $c_{\infty}^{-1}$. Along these branches numerical evidence for the existence of extensive regions of smooth steady transonic flow, with local Mach numbers as large as 1.276, is presented.

\section{Introduction}

In the spirit of Moore \& Pullin $(1987,1998)$ and Meiron, Moore \& Pullin (2000) we study the effect of compressibility on steady incompressible vortical solutions of Euler's equations in two dimensions. In particular, we seek continuous solution branches corresponding to smooth transonic flow, in contrast to Morawetz (1956, 1957, 1958) who showed that for flow past airfoils, solutions corresponding to smooth transonic flow, if they exist, must be isolated. By 'continuous solution branches' we mean that there exist admissable solutions in an arbitrarily small neighbourhood surrounding a particular solution in the space of the appropriate controlling parameters. In the present work we investigate the effects of compressibility on the Mallier \& Maslowe vortex. Our formulation and solution method is based on Meiron et al. (2000, henceforth referred to as MMP), who found small regions of smooth transonic flow in the parameter space of their compressible extension of the Stuart (1967) vortex.

Mallier \& Maslowe (1993, henceforth referred to as MM), proposed an exact nonlinear steady solution to the incompressible two-dimensional Euler equations, representing a periodic row of counter-rotating vortices. The periodic direction will be denoted $x$, with $y$ as the transverse coordinate. In dimensionless coordinates, for steady two-dimensional inviscid incompressible flow the velocity field, $\boldsymbol{u}(x, y)$, and vorticity, $\omega(x, y)$, may be represented using a stream function $\psi(x, y)$ :

$$
u=\frac{\partial \psi}{\partial y}, \quad v=-\frac{\partial \psi}{\partial x}, \quad \omega=-\nabla^{2} \psi
$$

The vorticity is chosen to be a function of $\psi$ alone, which satisfies the requirement 
that any steady solution of the incompressible Euler equations has constant vorticity along pathlines. The MM vortex corresponds to the choice

$$
\omega=-\nabla^{2} \psi=\frac{1}{2 \kappa^{2}} \sinh (2 \psi),
$$

where $1 \leqslant \kappa<\infty$ is a parameter defining the family. This is a form of the sinh-Gordon equation (the Stuart 1967 solution satisfies Liouville's equation) where the family of exact solutions is given by

$$
\psi_{0}(x, y)=\log \left(\frac{\kappa \cosh \left(\frac{\sqrt{\kappa^{2}-1}}{\kappa} y\right)-\sqrt{\kappa^{2}-1} \cos (x)}{\kappa \cosh \left(\frac{\sqrt{\kappa^{2}-1}}{\kappa} y\right)+\sqrt{\kappa^{2}-1} \cos (x)}\right) .
$$

By construction the flow is $2 \pi$-periodic in $x$, and the total circulation, $\Gamma_{v}$, associated with each vortex is independent of $\kappa$ and equal to $4 \pi$. As $\kappa$ increases the core size decreases, implying that the maximum vorticity must increase to conserve circulation. When $\kappa \rightarrow \infty, \psi_{0}$ can be shown to describe the potential flow produced by an array of counter-rotating point vortices, while $\kappa=1$ represents fluid at rest. The mass flow rate, $\epsilon$, between adjacent vortices can be defined in terms of $\kappa$,

$$
\epsilon=\psi_{0}(\pi, 0)-\psi_{0}(0,0)=4 \log \left(\kappa+\sqrt{\kappa^{2}-1}\right) \quad \Longrightarrow \quad \kappa=\cosh \left(\frac{1}{4} \epsilon\right),
$$

where $0 \leqslant \epsilon<\infty$.

\section{Compressible formulation and numerical solution}

\subsection{Formulation}

The governing equations are the compressible steady Euler equations in two space dimensions. We assume a calorically perfect gas with constant specific heats $c_{v}$ and $c_{p}$. Using * to denote a dimensional quantity, $\rho_{\infty}^{*}$ to denote the constant density as $|y| \rightarrow \infty$ and $l^{*}$ as a characteristic vortex length scale, the reference pressure is $p_{r}^{*}=u_{v}^{* 2} \rho_{\infty}^{*}$, with the characteristic vortex velocity defined as $u_{v}^{*}=\Gamma_{v}^{*} /\left(l^{*} \Gamma_{v}\right) . \Gamma_{v}$ is the dimensionless circulation of an individual vortex, which is to be held constant by construction. In the following, fluid quantities are made non-dimensional with respect to these reference values. The dimensionless speed of sound is $c_{\infty}=\left(\gamma p_{\infty} / \rho_{\infty}\right)^{1 / 2}$, where $p_{\infty}, \rho_{\infty}$ are the dimensionless pressure and density respectively as $|y| \rightarrow \infty$. Following MMP, the main idea is to write the steady Euler equations in terms of a stream function $\psi(x, y)$ defined by

$$
\rho u=\frac{\partial \psi}{\partial y}, \quad \rho v=-\frac{\partial \psi}{\partial x},
$$

and density, $\rho(x, y)$. For steady, plane compressible flow the $\psi-\omega$ relationship is

$$
\omega(x, y)=-\frac{1}{\rho} \nabla^{2} \psi+\frac{1}{\rho^{2}}(\nabla \psi \cdot \nabla \rho) .
$$

The entropy equation is satisfied by $S=S(\psi)$. This restricts the MMP formulation to shockless flow but does not preclude smooth transonic flow. Using the total enthalpy

$$
H=\frac{1}{2} \boldsymbol{u}^{2}+\frac{c_{\infty}^{2}}{(\gamma-1)} \frac{p}{\rho},
$$


and the ideal-gas equation of state, the momentum equation can be written as

$$
\boldsymbol{\omega} \times \boldsymbol{u}=-\nabla H+\frac{c_{\infty}^{2}}{\gamma(\gamma-1)} T \nabla S
$$

Closure requires specification of $S(\psi)$ and $H(\psi)$. We consider homentropic flow ( $S=$ const) and choose a functional form for $\omega$ which yields MM's solution as $c_{\infty}^{-1} \rightarrow 0$,

$$
\frac{\omega}{\rho}=\frac{\Gamma_{c}}{2 \cosh ^{2}\left(\frac{1}{4} \epsilon\right)} \sinh (2 \mu \psi)
$$

where $\mu$ and $\Gamma_{c}$ are parameters to be determined. When $c_{\infty}^{-1} \rightarrow 0$ we require $\mu \rightarrow 1$ and $\Gamma_{c} \rightarrow 1$ so that (2.5) reduces to (1.2). The parameter $\mu$ is necessary for wellposedness, while $\Gamma_{c}$ is a construction parameter introduced so that the circulation can be held constant. From (2.2), (2.3), (2.4), and (2.5), a pair of closed equations can be obtained:

$$
\begin{gathered}
\nabla^{2} \psi-\frac{1}{\rho}(\nabla \psi \cdot \nabla \rho)=-\frac{\rho^{2} \Gamma_{c}}{2 \cosh ^{2}\left(\frac{1}{4} \epsilon\right)} \sinh (2 \mu \psi), \\
\frac{1}{2 c_{\infty}^{2}}(\nabla \psi)^{2}+\frac{\rho^{2}\left(\rho^{\gamma-1}-1\right)}{\gamma-1}=\frac{\rho^{2} \Gamma_{c}}{4 \mu c_{\infty}^{2} \cosh ^{2}\left(\frac{1}{4} \epsilon\right)}(1-\cosh (2 \mu \psi)) .
\end{gathered}
$$

On the semi-infinite rectangle, $\mathscr{R}=\{(x, y): 0 \leqslant x \leqslant \pi, 0 \leqslant y<\infty\}$, the boundary conditions are

$$
\left.\begin{array}{rll}
\frac{\partial \psi}{\partial y}=0 \quad \text { on } & (y=0,0 \leqslant x \leqslant \pi), \\
\frac{\partial \psi}{\partial x}=0 & \text { on } \quad(x=0, \text { and } x=\pi, 0 \leqslant y \leqslant \infty), \\
\psi \rightarrow 0 & \text { as } \quad y \rightarrow \infty, \quad 0 \leqslant x \leqslant \pi, \\
\rho \rightarrow 1 & \text { as } \quad y \rightarrow \infty, \quad 0 \leqslant x \leqslant \pi,
\end{array}\right\}
$$

Characterizing solutions to (2.6)-(2.7) requires two further conditions. The first is a constraint on the total dimensionless circulation,

$$
\int_{\mathscr{R}}|\omega(x, y)| \mathrm{d} x \mathrm{~d} y \equiv \frac{\Gamma_{c}}{2 \cosh ^{2}\left(\frac{1}{4} \epsilon\right)} \int_{\mathscr{R}} \rho(x, y)|\sinh (2 \mu \psi(x, y))| \mathrm{d} x \mathrm{~d} y=4 \pi .
$$

The second is a constraint on $\psi$ and can be formulated in many ways. Here we define a two-parameter family of solutions by writing the mass flux in the form

$$
\psi(\pi, 0)-\psi(0,0)=\epsilon \Gamma_{c}, \quad \epsilon=4 \log \left(\kappa+\sqrt{\kappa^{2}-1}\right) .
$$

Comparing (2.10) with (1.4) shows that, when $c_{\infty}^{-1}>0, \epsilon$ is no longer the mass flux. We nonetheless retain $\epsilon$, or more particularly $\kappa$ defined by (2.10), as a convenient parameter characterizing solutions to (2.6)-(2.7). The unknowns are $\psi\left(x, y ; c_{\infty}^{-1}, \kappa\right)$, $\rho\left(x, y ; c_{\infty}^{-1}, \kappa\right), \mu\left(c_{\infty}^{-1}, \kappa\right)$, and $\Gamma_{c}\left(c_{\infty}^{-1}, \kappa\right)$. Hence on a branch along which $\kappa$ is held constant and $c_{\infty}^{-1}$ increases, the mass flux $\epsilon(\kappa) \times \Gamma_{c}\left(c_{\infty}^{-1}, \kappa\right)$ in general varies. We remark that different right-hand sides of (2.10) generate alternative families of solutions. The present continuation is of interest because it is found to produce solutions containing extensive regions of plane transonic flow. 


$\begin{array}{ccc}\kappa & \left|\Gamma_{c}\left(\kappa, c_{\infty}^{-1}=0\right)-1.0\right| & \left|\mu\left(\kappa, c_{\infty}^{-1}=0\right)-1.0\right| \\ 1.10 & 9.441 \times 10^{-6} & 9.430 \times 10^{-6} \\ 1.50 & 5.076 \times 10^{-6} & 5.075 \times 10^{-6} \\ 2.00 & 2.855 \times 10^{-6} & 2.854 \times 10^{-6} \\ 3.00 & 1.456 \times 10^{-6} & 1.272 \times 10^{-6} \\ 5.00 & 5.068 \times 10^{-5} & 1.288 \times 10^{-5}\end{array}$

TABLE 1. Error in the circulation constraint parameter, $\Gamma_{c}$, and the nonlinear eigenvalue, $\mu$ : $[M, N]=[60,60]$.

\subsection{Numerical method}

A spectral collocation method, similar to that used by MMP was employed to solve the boundary-value problem defined by equations (2.6), (2.6), (2.9) and (2.10). To start, $\psi$ and $\rho$ are written as

$$
\left.\begin{array}{l}
\psi=\sum_{n=0}^{N-1} \sum_{m=0}^{M-1} a_{m n} \cos (m x) \Phi_{2 n}(y), \\
\rho=1+\sum_{n=0}^{N-1} \sum_{m=0}^{M-1} b_{m n} \cos (m x) \Phi_{2 n}(y),
\end{array}\right\}
$$

where $a_{m n}$ and $b_{m n}$ are coefficients to be determined. The functions $\Phi_{n}(y)$ are basis functions, which decay as $y \rightarrow \infty$. The interval $0 \leqslant Y<1$ was stretched onto $0 \leqslant y<\infty$ using the algebraic stretching, $Y=y / \sqrt{\eta^{2}+y^{2}}$, where $\eta$ is the stretching parameter. The functions $\Phi_{n}(y)$ are combinations of Chebyschev polynomials satisfying the boundary conditions. Letting $\phi_{n}(y(Y))=T_{n}(Y)$, then $\Phi_{n}(y)=\phi_{0}(y)-\phi_{n+2}(y)$. The collocation points are $\left(x_{i}, y\left(Y_{j}\right)\right), i=1, \ldots M, j=1, \ldots N$, where $x_{i}$ are the zeros of $\cos (M x)$ between 0 and $\pi$, and $Y_{j}$ are the zeros of $T_{2 N}(Y)$ between 0 and 1. Equations (2.11) were substituted into each of (2.6) and (2.7) and satisfied at the collocation points. This combined with equation (2.9) and (2.10) yielded $2 \times M \times N+2$ nonlinear equations for the $2 \times M \times N+2$ unknowns $a_{m n}, b_{m n}, \mu$ and $\Gamma_{c}$. These equations were solved by a standard Newton method with analytical evaluation of the Jacobian, which was full. Numerical solutions reported have residuals less than $10^{-10}$.

\section{Compressible Mallier \& Maslowe vortex}

\subsection{Continuation to finite $c_{\infty}$}

To continue the MM vortex to finite $c_{\infty}, \kappa$ was fixed and a numerical solution obtained for $c_{\infty}^{-1}=0$ by using as an initial approximation a set of coefficients $a_{m n}$ calculated using equation (1.3), $b_{m n}=0, \mu=1$ and $\Gamma_{c}=1$. The spectral solutions reported use $[M, N]=[40,40],[60,60], \eta=1.5$. The accuracy of the method was tested by comparing numerical results with the analytical solution at $c_{\infty}^{-1}=0$, shown in table 1 .

Solutions were obtained with $\kappa=1.10,1.50,2.00,3.00,5.00$, over a range of $c_{\infty}$ varying from $c_{\infty}^{-1}=0$ to a maximum value, which will be addressed later. Figure 1 shows contour plots of $\psi_{0}$ and $\omega_{0}$ for the incompressible MM vortex at $\kappa=5.0$, and plots of $\psi, \omega, \rho$ and $\nabla \cdot \boldsymbol{u}$ for the compressible MM vortex at $\kappa=5.0$ and $c_{\infty}^{-1}=0.325$. There is a striking difference between the incompressible and compressible stream functions and vorticity. In particular, increasing compressibility increases the strain 

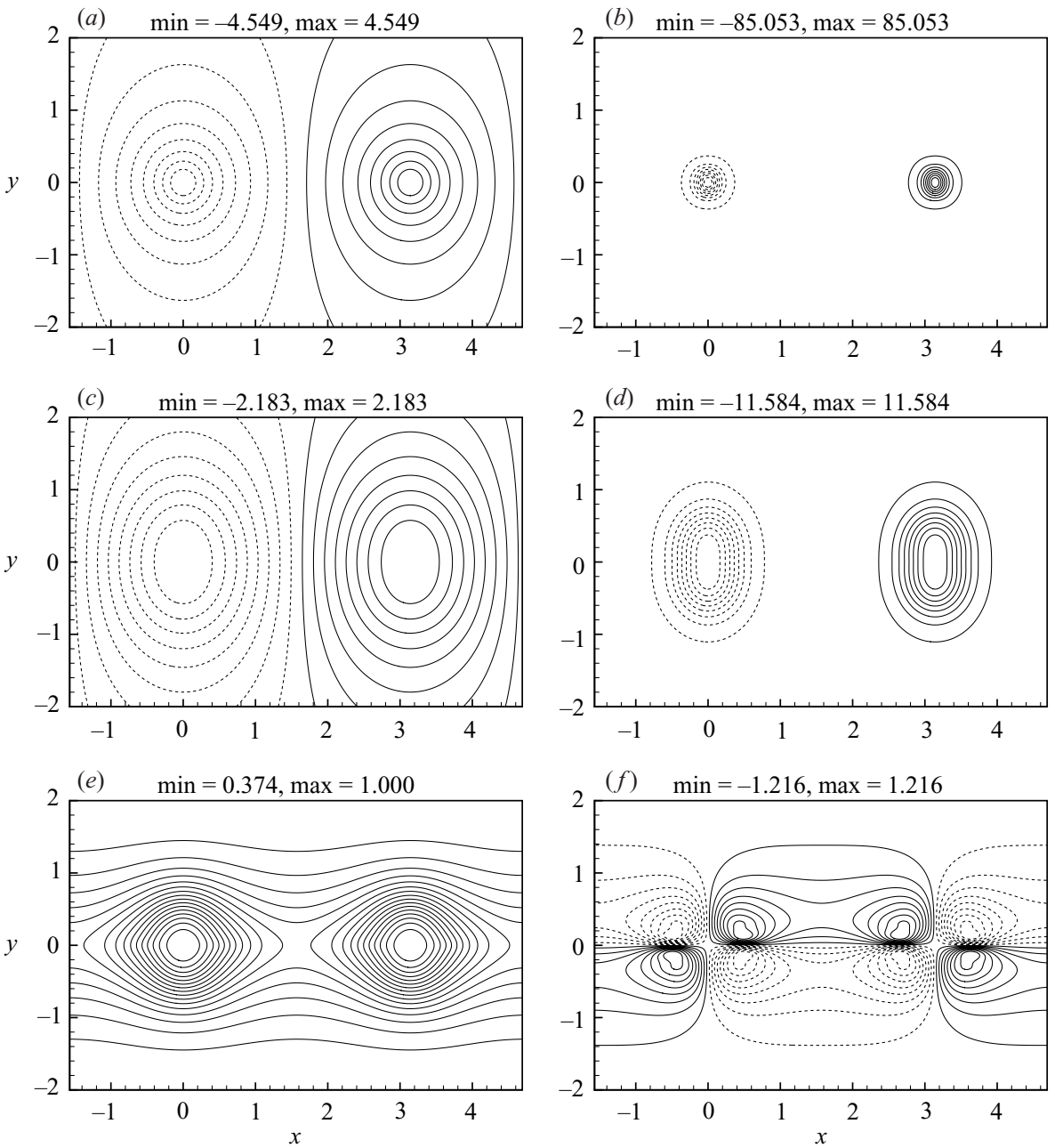

Figure 1. Incompressible MM vortex; $\kappa=5.0, c_{\infty}^{-1}=0$ : $(a)$ stream function, $(b)$ vorticity. Compressible MM vortex; $\kappa=5.0, c_{\infty}^{-1}=0.325:(c)$ stream function, $(d)$ vorticity, $(e)$ density, $(f)$ dilatation.

rate inside the vortex cores, which in turn stretches the vortices substantially in the transverse direction. Indeed, the ratio of minor to major axes increases by at least a factor of five from its incompressible value over the range of $\kappa$ investigated. The local Mach number, $M_{l}$, is defined as

$$
M_{l}=c_{\infty}^{-1}\left(\frac{(\partial \psi / \partial x)^{2}+(\partial \psi / \partial y)^{2}}{\rho^{\gamma+1}}\right)^{1 / 2} .
$$

The maximum Mach number, $M_{\max }$, occurs on the symmetry line, $y=0$, near the vortex core boundary where the vertical velocity is maximum, figure 2 . The overall effect of increasing compressibility on the MM vortex, at fixed $\kappa$, is shown in figures 3-5. Plotted are $\mu\left(c_{\infty}^{-1}, \kappa\right)$ and $\Gamma_{c}\left(c_{\infty}^{-1}, \kappa\right)$ in figures $3(a)$ and $3(b), 2 s(n \pi, 0) / \omega(n \pi, 0)$ and $M_{\max }$ in figures $4(a)$ and $4(b)$, where $s(n \pi, 0)$ is the strain rate at a vortex core, and finally $\rho(n \pi, 0)$ and $\omega(\pi, 0)$ in figures $5(a)$ and $5(b)$. 


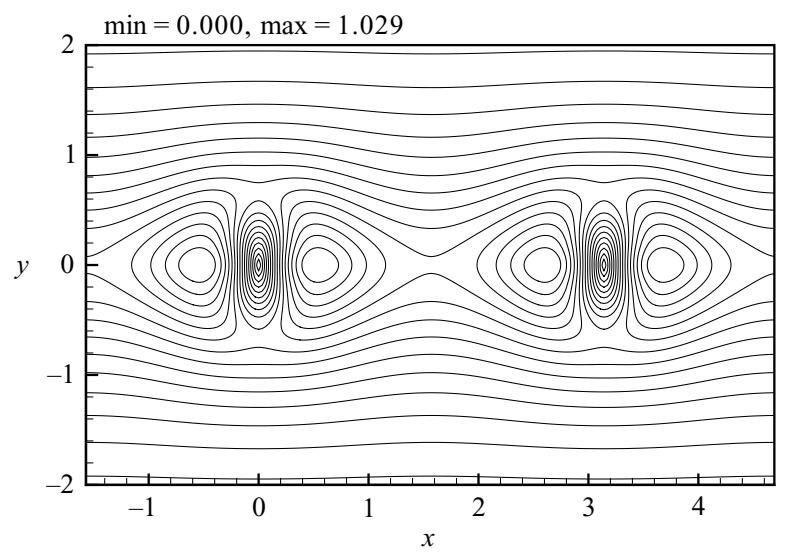

FiguRE 2. Contour of local Mach number, $M_{l} ; \kappa=5.00, c_{\infty}^{-1}=0.325$.
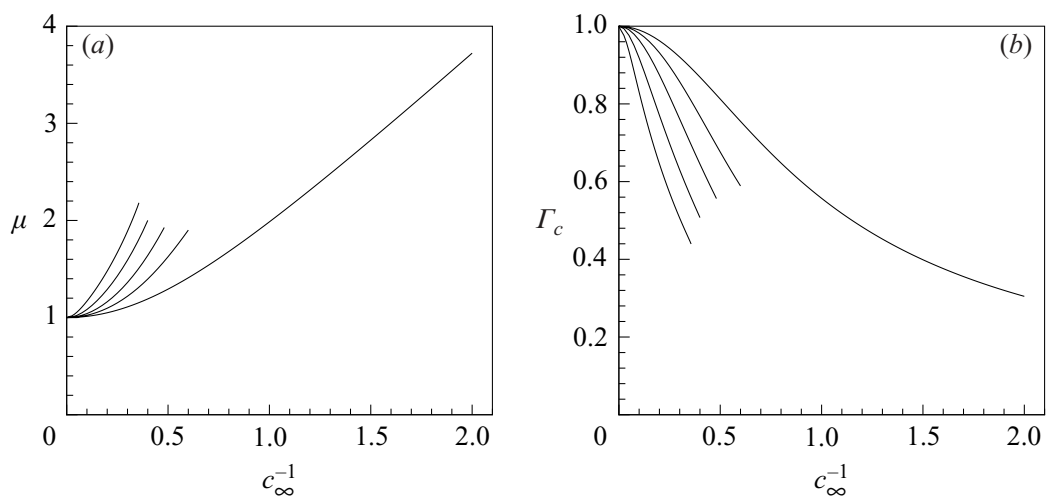

FiguRE 3. Solution parameters. (a) Nonlinear eigenvalue $\mu$ versus $c_{\infty}^{-1}$. (b) Circulation constraint $\Gamma_{c}$ versus $c_{\infty}^{-1} \cdot \kappa=1.10,1.50,2.00,3.00,5.00, \kappa$ increasing right to left.
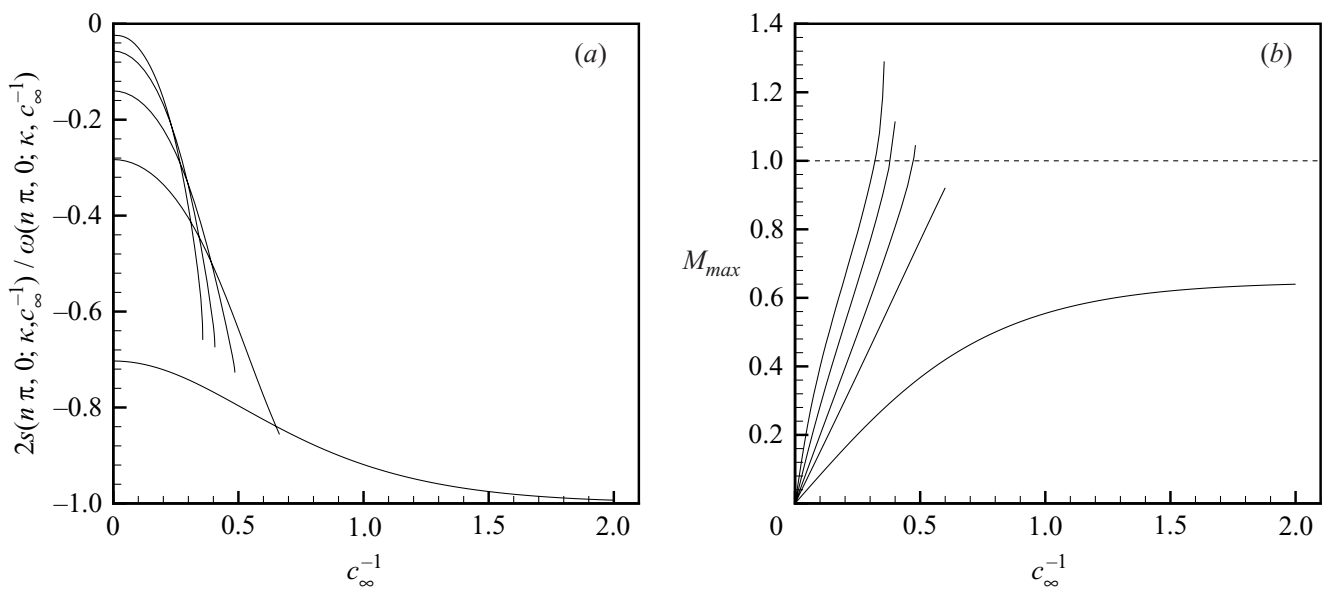

Figure 4. (a) Normalized strain versus $c_{\infty}^{-1}$. (b) $M_{\max }$ versus $c_{\infty}^{-1}$. Values of $\kappa$ as in figure 3, $\kappa$ increasing bottom to top in $(a)$, and right to left in $(b)$. 

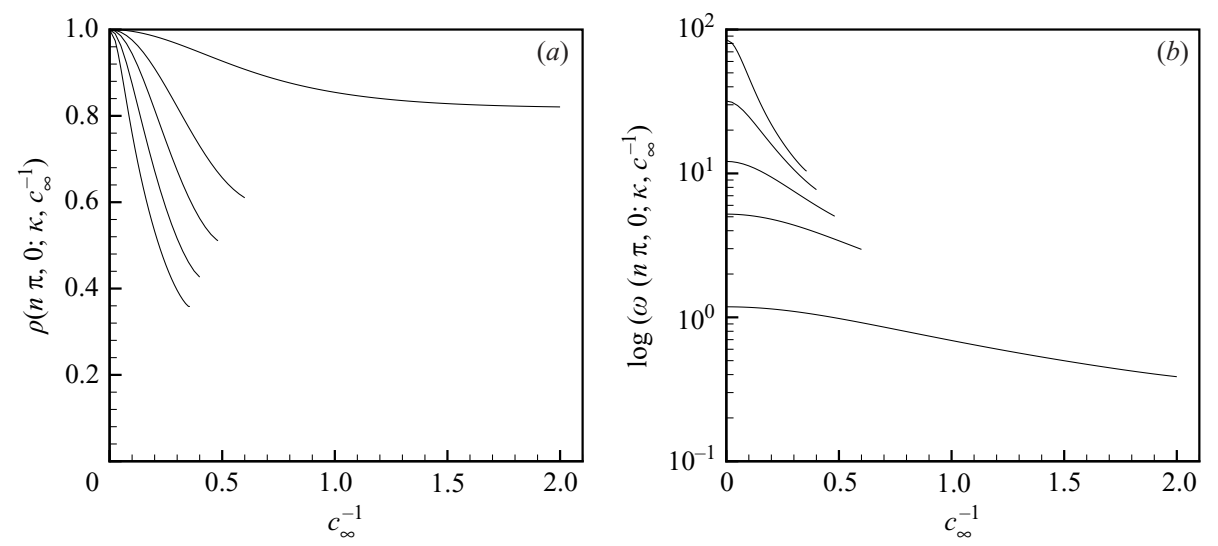

Figure 5. (a) Minimum density versus $c_{\infty}^{-1}$. (b) Maximum vorticity versus $c_{\infty}^{-1}$. Values of $\kappa$ as in figure 3 , increasing top to bottom in $(a)$ and bottom to top in $(b)$.

Increasing $c_{\infty}^{-1}$ decreases $\Gamma_{c}$, figure $3(b)$, which in turn decreases the effective mass flow rate between the vortex cores. This leads to a very large increase in the absolute value of the normalized strain, figure $4(a)$, and so the vortices are stretched substantially in the vertical direction. Figure 5(b) shows that the maximum value of vorticity, which is realized at the vortex cores, also decreases as $c_{\infty}^{-1}$ is increased. To maintain a constant circulation, the vortex cores become less compact, which causes the low-density region inside the vortex cores to extend into the high-velocity region between the cores, thereby increasing the maximum local Mach number.

Figure 4(b) shows that the onset of locally smooth supersonic flow depends on $\kappa$. At low vales of $\kappa$, the flow remains entirely subsonic, and the bound $\mid s(n \pi, 0) /$ $\frac{1}{2} \omega(n \pi, 0) \mid<1$, necessary for the streamlines to remain elliptical, is close to being violated. Indeed for $\kappa=1.10, c_{\infty}^{-1}=2.00, s(n \pi, 0) / \frac{1}{2} \omega(n \pi, 0)=-0.993$. In this regime $\epsilon$ is small, so that increasing compressibility causes the effective mass flux, $\epsilon \times \Gamma_{c}$, to tend to zero. The vortices become weak and less compact, so that this solution branch asymptotes to quiescent flow. For large values of $\kappa$ above a critical $c_{\infty}^{-1}$, the value of which depends on $\kappa$, the solutions admit the existence of smooth regions of supersonic flow.

\subsection{Spectral convergence}

To address the question of convergence, the decay of the coefficients $a_{m n}$ and $b_{m n}$ with respect to $m$ was examined. The magnitude of the coefficients was assumed to have an exponential form:

$$
\left|a_{m n}\right| \sim \exp \left(\alpha_{m}\left(n ; \kappa, c_{\infty}^{-1}\right) m\right), \quad\left|b_{m n}\right| \sim \exp \left(\beta_{m}\left(n ; \kappa, c_{\infty}^{-1}\right) m\right),
$$

with $n$ fixed. Exponential convergence is lost when any of the exponents pass through zero: $\alpha_{m} \geqslant 0$ or $\beta_{m} \geqslant 0$. Least-squares fits of $\log \left(\left|a_{m n}\right|\right)$ and $\log \left(\left|b_{m n}\right|\right)$ versus $m$ were made for several values of $n$. The slopes of these fits give estimates of $\alpha_{m}$ and $\beta_{m}$, thus yielding a criterion for when convergence is lost. In general, a solution branch is said to terminate when the Newton method fails to find converged solutions along a particular branch. Nevertheless, for small values of $\kappa$ the convergence of the spectral series is not lost along a particular branch. These branches asymptote to quiescent flow. Arc-length continuation in the space of all unknowns, with fourth-order extrapolation in arc-length to estimate the next approximation, was employed to 

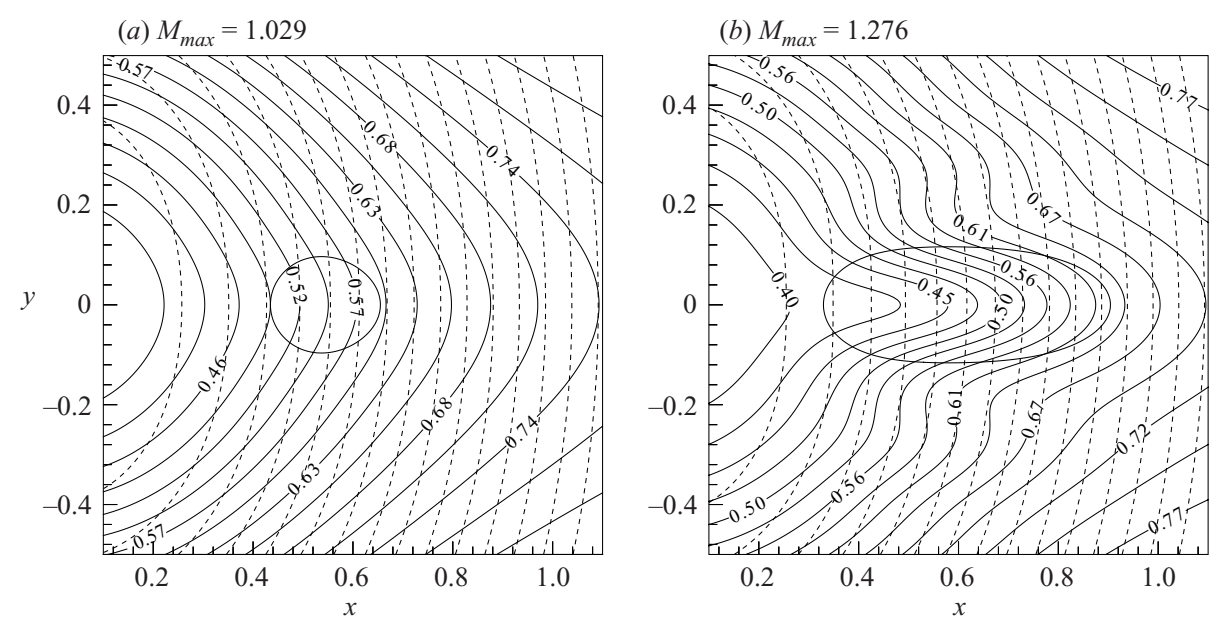

FIGURE 6. $\kappa=5.00$. Heavy solid line: sonic line, $M_{l}=1.0$. The flow contained in this region is supersonic. Dashed contours: stream function. Solid, labelled contours: density. $(a) c_{\infty}^{-1}=0.325$. (b) $c_{\infty}^{-1}=0.342$.

ensure that branch termination was genuine, and not a result of continuing in $c_{\infty}^{-1}$. Following MMP we interpret branch termination as incipient shocklet formation, in which case equations (2.6) and (2.7) are no longer valid because entropy cannot then be single valued on a spatially periodic domain.

\subsection{Transonic flow}

Large ranges of $c_{\infty}^{-1}$ and $\kappa$ were found which yield solutions showing smooth regions of supersonic flow, figure $4(b)$. For $\kappa=5.0$ the numerical solutions show the onset of transonic flow for $c_{\infty}^{-1}$ in the range 0.319 to 0.342 . Three-point interpolation gives $M_{\max }=1.00$ at $c_{\infty}^{-1}=0.3187$. At $c_{\infty}^{-1}=0.342, M_{\max }=1.276$. For this value of $\kappa$ no solutions were found at greater $c_{\infty}^{-1}$ displaying exponential decay of the spectral coefficients.

Figure 6 shows contours of $\psi$ and $\rho$ windowed on the supersonic region, which is of sizable extent. The low vortex core density bulges into the supersonic region, so that on following a streamline we see a substantial decrease in density, followed by a matching increase to preserve symmetry about the $x$-axis. Similar plots of $\nabla \cdot \boldsymbol{u}$ show that these large density gradients cause the magnitude of the dilatation to increase, and show steep gradients inside the sonic bubble. After MMP, we postulate that this may indicate that at larger values of $c_{\infty}^{-1}$ incipient weak shocks would form, which would cause convergence failure of the cosine series. The bulging core density field is a direct result of allowing the mass flux to vary along a solution branch. This allows regions of low sound speed and high velocity to intersect, thereby producing supersonic values of the local Mach number. The rotational character of the present Mallier \& Maslowe vortex flow may explain the apparent non-conformity with the results of Morawetz $(1956,1957,1958)$.

\section{Concluding remarks}

Spectrally accurate numerical solutions to the steady compressible two-dimensional Euler equations, representing a continuation to finite $c_{\infty}$ of the Mallier \& Maslowe (1993) vortex, have been obtained. As in Meiron et al. (2000) an eigenvalue was 
introduced into the vorticity-density-stream-function relationship to continue the MM vortex to the compressible regime. Unexpectedly, it was also required that an extra parameter, $\Gamma_{c}$, be intercalated so that the circulation constraint could be enforced. Its value was determined as part of the overall solution, and was seen to vary with both mass flux between the vortex cores, and the sound speed at infinity. Increasing compressibility was seen to appreciably increase the aspect ratio of the vortices, while reducing the minimum value of density at the vortex core. Numerical evidence was presented for the existence of a substantial range of free-stream sound speeds over which, at fixed $\kappa$, the solution corresponded to smooth transonic flow. For $\kappa=5.00$, regions of shock-free supersonic flow were found between $0.319<c_{\infty}^{-1}<0.342$. Branch termination is attributed to the large vertical dilatational gradients inside the locally supersonic region. This may indicate the incipient formation of weak shocks which cannot be accommodated within our formulation. The present study has focused on the existence of solution branches containing regions of smooth transonic flow. The stability of these steady solutions is unknown although we note that Julien, Chomaz \& Lasheras (2002) have shown that the limiting incompressible MM vortex admits several classical instability modes.

The notable feature of the present class of solutions is the surprisingly large local Mach numbers reached prior to branch termination. We remark that this homentropic continuation of the MM vortex to the compressible regime is not unique. Homentropic solution branches may be constructed with very different properties to those reported here. A formulation where the mass flux was held constant along a solution branch was also investigated, that is, replacing the right-hand side of (2.10) by $\epsilon$. With these equations the vortices became more intense and compact as $c_{\infty}^{-1}$ was increased. At fixed mass flux all solution branches followed were found to exhibit small regions of locally supersonic flow prior to branch termination but the maximum local Mach numbers were substantially smaller and closer to unity than those maxima found for the homentropic continuation described in detail here. Additionally, homenthalpic continuations may be constructed but these have not been investigated in detail.

Finally, it may be possible to formulate interesting variations on the compressible MM vortex proposed here, for example bifurcation to solutions which enable the stagnation points at the vortex cores to become hyperbolic, or formulations for which symmetry about the $x$-axis is not enforced. A possible bifurcation to a non-symmetric states may admit larger regions of locally supersonic flow before solution branch termination than those found here.

This work was supported by the Advanced Simulation Computing program (ASC) under subcontract no. B523297 of DOE contract W-7405-ENG-48.

\section{REFERENCES}

Julien, S., Chomaz, J.-M. \& Lasheras, J. 2002 Three-dimensional stability of periodic arrays of counter-rotating vortices. Phys. Fluids 14, 732-743.

Mallier, R. \& Maslowe, S. 1993 A row of counter-rotating vortices. Phys. Fluids A 5, 1074-1075 (referred to herein as MM).

Meiron, D. I., Moore, D. W. \& Pullin, D. I. 2000 On steady compressible flows with compact vorticity; the compressible stuart vortex. J. Fluid Mech. 409, 29-49 (referred to herein as MMP).

Moore, D. W. \& Pullin, D. I. 1987 The compressible vortex pair. J. Fluid Mech. 185, 171-204.

Moore, D. W. \& Pullin, D. I. 1998 On steady compressible flows with compact vorticity; the compressible hill's spherical vortex. J. Fluid Mech. 374, 285-303. 
Morawetz, C. S. 1956 On the non-existence of continuous transonic flow past airfoils. I. Commun. Pure Appl. Maths 9, 45-68.

Morawetz, C. S. 1957 On the non-existence of continuous transonic flow past airfoils. II. Commun. Pure Appl. Maths 10, 107-131.

Morawetz, C. S. 1958 On the non-existence of continuous transonic flow past airfoils. III. Commun. Pure Appl. Maths 11, 129-144.

StUART, J. 1967 On finite amplitude oscillations in laminar mixing layers. J. Fluid Mech. 29, 417-440. 